\title{
THE FIRST INTERVAL OF STABILITY OF A PERIODIC EQUATION OF DUFFING TYPE
}

\author{
RAFAEL ORTEGA
}

(Communicated by Charles Pugh)

\begin{abstract}
Consider the differential equation of a nonlinear oscillator with linear friction and a $T$-periodic external force. We find optimal bounds on the derivative of the restoring force in order to obtain a unique $T$-periodic solution that is asymptotically stable.
\end{abstract}

Consider the differential equation

$$
x^{\prime \prime}+c x^{\prime}+g(x)=p(t)
$$

where $c>0$ is a fixed constant, $p$ is $T$-periodic, $g$ and $p$ are sufficiently smooth and satisfy

$$
0<g^{\prime}(x)<b \text { for each } x \in \mathbb{R}(b>0)
$$

and

$$
g(-\infty)<(1 / T) \int_{0}^{T} p(t) d t<g(+\infty)
$$

Under these assumptions, $(*)$ is a dissipative system (see $[9$, p. 51$],[11$, p. 71$])$ and, in particular, it has at least one $T$-periodic solution. When $b$ is small this periodic solution is unique and globally asymptotically stable; however, when $b$ becomes large, one can expect that for certain forcing terms $p(t)$ the attractor set will have a more complex structure so that unstable $T$-periodic solutions as well as subharmonic solutions can appear. In this paper we obtain the optimal condition on $b$ in order to guarantee the existence of a unique $T$-periodic solution that is (locally) asymptotically stable when $(\mathbf{H})$ holds.

Lazer and McKenna have already considered this problem in [3] when $(\mathbf{H})$ is replaced by the condition

$$
a \leq g^{\prime}(x) \leq b \text { for each } x \in \mathbb{R}(0<a<b) .
$$

They obtained sufficient conditions on $a$ and $b$ for the existence of a unique $T$-periodic solution that is asymptotically stable.

The problem that we have posed can be reduced to the linear question: to find the optimal $b>0$ such that there exist no skew-periodic solutions in the class of linear equations

$$
(* *) \quad y^{\prime \prime}+c y^{\prime}+\alpha(t) y=0 \quad(0 \leq \alpha(t)<b, t \in \mathbb{R}) .
$$

Received by the editors January 31, 1991.

1991 Mathematics Subject Classification. Primary 34C25, 34D20. 
For Hill's equation $(c=0)$ the analogous question is solved by comparison with the constant case $\left(\alpha=\right.$ constant) and, in fact, $b=(\pi / T)^{2}$ (see [1] or [5, p. 68]). For $c>0 \quad(* *)$ has not skew-periodic solutions for any constant $\alpha$, and we shall find that the role of model equation is now played by a certain equation with piecewise constant coefficients. This equation was already studied by Meissner in 1919 when $c=0$ (see [5, p. 115]). The relevance of this equation will come up from the use of the Maximal Principle of Potryangin. Following some ideas from [2] the skew-periodic problem for $(* *)$ is formulated as a problem in control theory and the optimal controllers are switching functions.

\section{MAIN THEOREMS}

In this section we consider the equation

$$
x^{\prime \prime}+c x^{\prime}+g(t, x)=p(t)
$$

where $g \in C(\mathbb{R} / T \mathbb{Z} \times \mathbb{R}), p \in C(\mathbb{R} / T \mathbb{Z})$. This equation is more general than $(*)$, and it will be assumed that the partial derivative $\partial_{x} g(t, x)$ is defined and continuous and satisfies

$$
0<\partial_{x} g(t, x)<b \text { for each }(t, x) \in \mathbb{R} \times \mathbb{R} .
$$

Also,

$$
\int_{0}^{T} g(t,-\infty) d t<\int_{0}^{T} p(t) d t<\int_{0}^{T} g(t,+\infty) d t
$$

Our first result gives a sharp estimate on $b$ (independent of the size of the period $T$ ) in order to guarantee that (1.1) has a unique $T$-periodic solution that is asymptotically stable. First we consider the equation in $\mathbb{R}$,

$$
\zeta \ln \left[\left(1+\zeta^{2}\right) / 4\right]=2 \arccos \left[-\left(1+\zeta^{2}\right)^{-1 / 2}\right], \quad \zeta \in \mathbb{R} .
$$

(Here, arccos: $[-1,1] \rightarrow[0, \pi]$.) It is easy to prove that $(1.4)$ has a unique positive root, denoted by $\zeta_{0}$. A computation shows that $\zeta_{0}=3.34354 \ldots$.

Theorem I. Assume that (1.2), (1.3) hold and

$$
b \leq\left(1+\zeta_{0}^{2}\right) c^{2} / 4
$$

Then (1.1) has a unique T-periodic solution that is asymptotically stable.

Remark. Assume that $g=g(x)$ does not depend on $t$. Then (1.1) is dissipative and the results in [10] together with some computations in [8] imply that this periodic solution is globally attracting if $b<c^{2} / 4$. We do not know if this is also true when only (1.5) is assumed.

As already mentioned, (1.5) is sharp. The next result will show that when it does not hold, there exist certain periods for which instability or nonuniqueness may occur. We now define a plane curve that plays an important role in the determination of the critical periods. For each $b>c^{2} / 4$ let $\mathbf{C}_{b}$ be the set of points $(x, y) \in \mathbb{R}_{+} \times \mathbb{R}_{+}$satisfying $J_{b}(x, y)=0$. Here,

$$
\begin{array}{r}
J_{b}(x, y)=2 \cos \delta x \operatorname{ch}(c y / 2)+\gamma \sin \delta x \operatorname{sh}(c y / 2)+2 \operatorname{ch}[c(x+y) / 2], \\
\text { with } \delta=\left[b-\left(c^{2} / 4\right)\right]^{1 / 2}, \gamma=(c / 2 \delta)-(2 \delta / c) .
\end{array}
$$

It will be proved that $\mathrm{C}_{b}$ is nonempty as soon as (1.5) does not hold (see Lemma 3.2 below). Typically $\mathbf{C}_{b}$ has a finite number of branches as described in Figure 1 . 


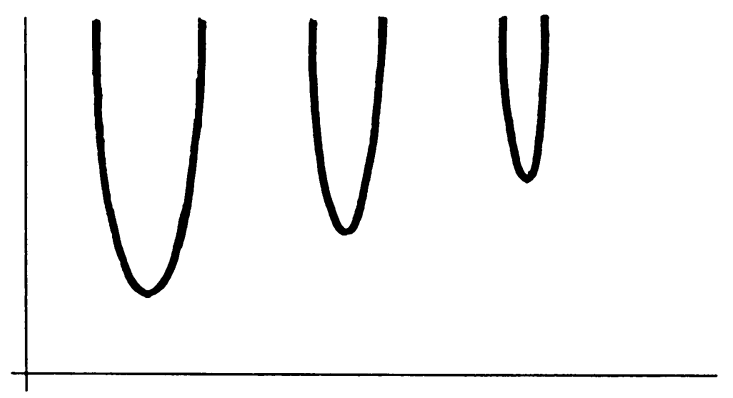

FIGURE 1

Associated with this curve we define

$$
\tau(b)=\min \left\{x+y \mid(x, y) \in \mathbf{C}_{b}\right\} .
$$

Remark that the effective computation of $\tau(b)$ reduces to an optimization problem for two variables with one constraint. Also, $\tau$ is strictly decreasing as a function of $b$.

In the next result we shall need the additional assumption

$$
\begin{gathered}
g \text { is independent of } t \text { and } \inf \left\{g^{\prime}(x) ; x \in \mathbb{R}\right\}=0, \\
\sup \left\{g^{\prime}(x) ; x \in \mathbb{R}\right\}=b .
\end{gathered}
$$

Theorem II. Assume that (1.2), (1.3) hold and $b>\left(1+\zeta_{0}^{2}\right) c^{2} / 4$. Then

(i) If $T \leq \tau(b)$ the conclusion of Theorem $\mathrm{I}$ is still true.

(ii) If $T>\tau(b)$ and (1.7) hold there exists some $p \in \mathbf{C}(\mathbb{R} / T \mathbb{Z})$ satisfying (1.3) and such that (1.1) has an unstable T-periodic solution and at least one second order subharmonic solution.

Remarks. 1. In the assumptions of Theorem I or Theorem II(i) it will follow from the proofs that $(1.1)$ has no subharmonic solutions of period $2 T$.

2. If $T<\tau(b)+\delta$, with $\delta$ given by (1.6), it can be proved that (1.1) has still a unique $T$-periodic solution (see Lemma 5.2).

\section{A SKEW PERIODIC PROBLEM}

Consider the linear equation

$$
y^{\prime \prime}+c y^{\prime}+\alpha(t) y=0, \quad \alpha \in L^{\infty}(\mathbb{R} / T \mathbb{Z}) .
$$

We are interested in skew-periodic solutions. A nontrivial solution of $(2.1)$ is called skew-periodic (or antiperiodic) if it satisfies $y(t+T)=-y(t)$ for each $t \in \mathbb{R}$. Since the equation is linear, every second order subharmonic solution must be skew-periodic.

Associated with (2.1) is the system

$$
x^{\prime}=A(t) x, \quad A(t)=\left(\begin{array}{cc}
0 & 1 \\
-\alpha(t) & -c
\end{array}\right) .
$$

Let $X(t)$ denote the matrix solution of (2.2) satisfying $X(0)=I$. The discriminant of $(2.1)$, denoted by $\Delta[\alpha]$, is defined as the trace of $X(T)$. It follows from Floquet theory that the existence of skew-periodic solutions of $(2.1)$ is equivalent to

$$
\Delta[\alpha]=-[1+\exp (-c T)] .
$$

Later we shall use the following continuity property of $\Delta$. 
Lemma 2.1. Let $\left\{\alpha_{n}\right\}$ be a bounded sequence in $L^{\infty}(\mathbb{R} / T \mathbb{Z})$ converging to $\alpha \in$ $L^{\infty}(\mathbb{R} / T \mathbb{Z})$ in the weak ${ }^{*}$ sense. Then $\lim \Delta\left[\alpha_{n}\right]=\Delta[\alpha]$.

Proof. Let $X_{n}(t)$ be the matrix solution of (2.2) for $\alpha_{n}$ with $X_{n}(t)=I+$ $\int_{0}^{t} A_{n}(s) X_{n}(s) d s, t \in \mathbb{R}$. It follows from the Gronwall lemma that $\left\{X_{n}(t)\right\}$ is uniformly bounded and equicontinuous on $[0, T]$. For each subsequence $X_{k} \rightarrow X$ uniformly in $[0, T], A_{k} X_{k} \rightarrow A X$ in $L^{\infty}$-weak*. Then $X$ is the solution matrix of (2.2) with $X(0)=I$. The uniqueness of the matrix and the Ascoli theorem imply that $X_{n} \rightarrow X$ uniformly in $[0, T]$. In particular, trace $X_{n}(T) \rightarrow \operatorname{trace} X(T)$.

\section{MEISSNER'S EQUATION}

Given $\sigma \in(0, T)$ define the periodic switching function

$$
s_{\sigma} \in L^{\infty}(\mathbb{R} / T \mathbb{Z}), \quad s_{\sigma}(t)= \begin{cases}1, & t \in(0, \sigma), \\ 0, & t \in(\sigma, T),\end{cases}
$$

and for each $b>0$, consider the equation

$$
y^{\prime \prime}+c y^{\prime}+b s_{\sigma}(t) y=0 \text {. }
$$

This equation can be explicitly integrated. For $b \leq c^{2} / 4$ it is always asymptotically stable while for $b>c^{2} / 4$ the discriminant is

$$
\Delta\left[b s_{\sigma}\right]=\exp (-c T / 2)[2 \cos \delta \sigma \operatorname{ch}(c(T-\sigma) / 2)+\gamma \sin \delta \sigma \operatorname{sh}(c(T-\sigma) / 2)]
$$

with $\delta$ and $\gamma$ given by (1.6).

The definition of $\mathbf{C}_{b}$ together with (2.3) implies that (3.1) has skew-periodic solutions if and only if $(\sigma, T-\sigma) \in C_{b}$. This remark leads to the following result.

Proposition 3.1. (i) If (1.5) holds or $T<\tau(b)$, then (3.1) has no skew-periodic solutions. (ii) If (1.5) does not hold and $T \geq \tau(b)$, there exists $\sigma \in(0, T)$ such that (3.1) has skew-periodic solutions. Moreover, if $T>\tau(b)$, there exists $\tilde{\sigma} \in(0, T)$ such that (3.1) is inversely unstable.

Remark. (2.1) is inversely unstable if the Floquet multipliers satisfy $\mu_{1}<-1<$ $\mu_{2}<0$ or, equivalently, $\Delta[\alpha]<-(1+\exp (-c T))$.

The proof is reduced to the obtention of the following properties of $J_{b}$.

Lemma 3.2. (i) $J_{b}(x, y)>0 \quad \forall(x, y) \in \mathbb{R}_{+} \times \mathbb{R}_{+} \Leftrightarrow b \leq\left(1+\zeta_{0}^{2}\right) c^{2} / 4$.

Let $\left(x_{0}, y_{0}\right) \in \mathbb{R}_{+} \times \mathbb{R}_{+}$be such that $J_{b}\left(x_{0}, y_{0}\right)=0$; then

(ii) $J_{b}\left(x_{0}, y\right)<0$ for each $y>y_{0}$,

(iii) $\forall t>x_{0}+y_{0} \exists\left(x_{t}, y_{t}\right), x_{t}+y_{t}=t, J_{b}\left(x_{t}, y_{t}\right)=0$.

Proof. (i) $J_{b}=0$ can be solved with respect to $y$ to obtain

$$
y=(2 / c) \operatorname{coth}^{-1}[-(y \sin \delta x+2 \operatorname{sh}(c x / 2)) /(2 \cos \delta x+2 \operatorname{ch}(c x / 2)] .
$$

Then $J_{b}>0$ on $\mathbb{R}_{+} \times \mathbb{R}_{+}$if and only if $f_{b}(x) \geq 0$ for each $x \in(0, \infty)$ with $f_{b}(x)=2 \cos \delta x+\gamma \sin \delta x+2 \exp (c x / 2)$. Define $m(b)=\inf _{[0, \infty)} f_{b}$. Since $m(b)$ is reached at some point in the interval $[0,2 \pi / \delta]$, it is easily proved that $m$ is continuous with respect to $b$. Since $m(+\infty)=-\infty$ and $m(b)>0$ as $b \downarrow c^{2} / 4$, there exists $b_{0}$ such that $m\left(b_{0}\right)=0$. For $b=b_{0}$ there exists $x \in$ $[0,2 \pi / \delta)$ such that $f_{b}^{\prime}(x)=f_{b}(x)=0$, leading to $2 \cos \delta x+(c / \delta) \sin \delta x=0$. 
In consequence, $v=(\cos \delta x, \sin \delta x)$ lies either on the second or the fourth quadrant. If $v$ is in the fourth quadrant, $f_{b}(x-(\pi / 2 \delta))<f_{b}(x)=0$, a contradiction with the definition of $b=b_{0}$. Therefore $v$ is in the second quadrant and, again using $f_{b}(x)=0$, a computation shows that $2 \delta / c$ satisfies (1.4) and $b_{0}=\left(1+\zeta_{0}^{2}\right) c^{2} / 4$. Since $\gamma$ is negative for $b \geq b_{0}$, the definition of $f_{b}$ proves that $m(b)<m\left(b_{0}\right)$ if $b>b_{0}$, finishing the proof.

(ii) The function $J_{b}\left(x_{0}, \cdot\right)$ is a solution of $z^{\prime \prime}-\left(c^{2} / 4\right) z=0$ and therefore has at most one simple zero.

(iii) Let $y_{1}>0$ be such that $x_{0}+y_{1}=t$. Then $J_{b}\left(x_{0}, y_{1}\right)<0$ by (ii). Since $J_{b}(t, 0)>0$ there exists $\lambda \in(0,1)$ such that $J_{b}\left(\lambda t+(1-\lambda) x_{0},(1-\lambda) y_{1}\right)=0$.

\section{A CONSEQUENCE OF THE MAXIMAL PRINCIPLE}

We shall prove the following

Proposition 4.1. Assume that for some $\alpha \in L^{\infty}(\mathbb{R} / T \mathbb{Z})$ satisfying

$$
0 \leq \alpha(t) \leq b \text { a.e. } t \in \mathbb{R},
$$

equation (2.1) has skew-periodic solutions. Then there exists $\sigma \in(0, T)$ such that (3.1) has also skew-periodic solutions.

Before the proof we point out some facts on the boundary value problem

$$
\begin{array}{lll}
y^{\prime \prime}+c y^{\prime}+u(t) y=0 & \text { a.e. } t \in(0, \tau)\left(u \in L^{\infty}(0, \tau)\right), \\
y(0)=y(\tau)=0, & y^{\prime}(0)=-y^{\prime}(\tau)=1 & (\tau>0) .
\end{array}
$$

Lemma 4.2. Assume that (4.2) has a solution satisfying $y(0)=y(\tau)=0,0<$ $y^{\prime}(0) \leq-y^{\prime}(\tau)$ when $u=u_{1}$, for some $u_{1} \in L^{\infty}(0, \tau)$. Then there exists $u_{2} \in L^{\infty}(0, \tau)$ with ess inf $u_{1} \leq u_{2}(t) \leq \operatorname{ess} \sup u_{1}$, a.e. $t \in(0, \tau)$, and such that (4.2), (4.3) is solvable for $u=u_{2}$.

Proof. Let $u_{1}^{\#} \in L^{\infty}(\mathbb{R} / \tau \mathbb{Z})$ be the periodic extension of $u_{1}$. The solution $y(t)$ is extended to $\mathbb{R}$ as a solution of $(2.1)$ for $\alpha=u_{1}^{\#}$ in such a way that $y(t+\tau)=\mu y(t), t \in \mathbb{R}$, with $\mu=y^{\prime}(\tau) / y^{\prime}(0)$. In consequence $\mu<-1$ is a Floquet multiplier and $\Delta\left[u_{1}^{\#}\right] \leq-(1+\exp (-c \tau))$. The continuity of $\Delta$ implies the existence of $\lambda \in[0,1]$ such that $\Delta\left[\lambda u_{1}^{\#}+(1-\lambda) \bar{m}\right]=-(1+\exp (-c \tau))$ with $\bar{m}=\left(\right.$ ess sup $\left.u_{1}+\operatorname{ess} \inf u_{1}\right) / 2$. (Recall $\left.\Delta[\bar{m}]>-(1+\exp (-c \tau))\right)$. Then $(2.1)$ has skew-periodic solutions (period $\tau$ ) for $\alpha=\lambda_{21}^{\#}+(1-\lambda) \bar{m}$, and it follows that (4.2) and (4.3) is solvable for some time translation of $\alpha$.

Proof of Proposition 4.1. We shall use the language and methods of control theory (see [4]) in a proof inspired by [2]. Consider the control process

$$
x^{\prime}=A[u] x, \quad x=\operatorname{col}\left(x_{1}, x_{2}\right), \quad A[u]=\left(\begin{array}{cc}
0 & 1 \\
-u & -c
\end{array}\right),
$$

with initial state $X_{0}=\operatorname{col}(0,1)$ and target state $X_{1}=\operatorname{col}(0,-1)$. The class of admissible controllers is $\mathbf{U}=\left\{u \in L^{\infty}(0, \tau) \mid \tau>0,0 \leq u(t) \leq b\right.$ a.e. $t \in(0, \tau)\}$. Notice that the attainability of $X_{1}$ is equivalent to the solvability of (4.2), (4.3).

By assumption there exists $\alpha \in L^{\infty}(\mathbb{R} / T \mathbb{Z})$ satisfying (4.1) and $y(t)$ skewperiodic solution of (2.1). Let $t_{0} \in[0, T)$ be such that $y\left(t_{0}\right)=y\left(t_{0}+T\right)=$ 0 . It is not restrictive to assume $y^{\prime}\left(t_{0}\right)=-y^{\prime}\left(t_{0}+T\right)=1$. The function 
$u(t)=\alpha\left(t+t_{0}\right)$ is an admissible control and the corresponding response $x(t)=$ $\operatorname{col}\left(y\left(t+t_{0}\right), y^{\prime}\left(t+t_{0}\right)\right)$ allows to attain the target set after time $T$. The existence of an optimal control for the associated time-optimal control problem follows from [4]. Let $u^{*}(t)$ be the optimal control defined in $\left(0, T^{*}\right)$ with $0<T^{*} \leq T$ and $x^{*}(t)$ the corresponding response. We divide the rest of the proof in three steps.

Step 1. $x_{1}^{*}(t)>0$ for each $t \in\left(0, T^{*}\right)$. Since $x_{1}^{*}$ is a solution of (4.2) for $u=u^{*}$, the zeros of $x_{1}^{*}$ are simple. By a contradiction argument assume that $x_{1}^{*}$ changes sign. There exist consecutive zeros $0<\tau_{1} \cdots<\tau_{2 n}<T^{*}, n \geq 1$, with $(-1)^{i}(d / d t) x_{1}^{*}\left(\tau_{i}\right)>0$. From $(d / d t) x_{1}^{*}(0)=-(d / d t) x_{1}^{*}\left(T^{*}\right)=1$ we infer the existence of $i, 0 \leq i \leq 2 n$, such that $\left|(d / d t) x_{1}^{*}\left(\tau_{i}\right)\right| \leq\left|(d / d t) x_{1}^{*}\left(\tau_{i+1}\right)\right|$. After the time translation $t \rightarrow t-\tau_{i}$ we apply Lemma 4.2 with $\tau=\tau_{i+1}-\tau_{i}$ and find that the target set can be attained after time $\tau$, a contradiction with the optimality of $T^{*}$

Step 2. Application of the maximal principle. The Hamiltonian function is given by $H(\eta, x, u)=\eta \cdot A[u] x=\left(\eta_{1}-c \eta_{2}\right) x_{2}-u \eta_{2} x_{1}$ and $M(\eta, x)=$ $\max _{0 \leq u \leq b} H(\eta, x, u)=\left(\eta_{1}-c \eta_{2}\right) x_{2}+b\left(\eta_{2} x_{1}\right)^{-}$. Then $H\left(\eta^{*}(t), x^{*}(t), u^{*}(t)\right)=$ $M\left(\eta^{*}(t), x^{*}(t)\right)$ a.e. $t \in\left(0, T^{*}\right)$, where $\eta^{*}=\left(\eta_{1}^{*}, \eta_{2}^{*}\right)$ is a nontrivial solution of $\eta^{\prime}=-A\left[u^{*}(t)\right]^{\tau} \eta$. In consequence

$$
u^{*}(t)= \begin{cases}0 & \text { if } \eta_{2}^{*}(t)>0 \\ b & \text { if } \eta_{2}^{*}(t)<0 .\end{cases}
$$

Step 3. Since $\eta_{2}^{*}(t)$ and $x_{1}^{*}(t)$ are solutions of adjoint equations, it follows from Step 1 that $\eta_{2}^{*}(t)$ has at most one zero in $\left(0, T^{*}\right)$. Therefore $u^{*}$ has at most one jump in $\left(0, T^{*}\right)$ and we can extend $u^{*}$ periodically so that $x_{1}^{*}(t)$ is a skew-periodic solution (of period $T^{*}$ ). Perhaps after a time-translation we see that $u^{*} \in L^{\infty}\left(\mathbb{R} / T^{*} \mathbb{Z}\right)$ is a switching function of the class considered in $\S 2$. An application of Proposition 3.1 shows that $T \geq T^{*} \geq \tau(b)$, concluding the proof.

\section{PROOFS OF THE MAIN THEOREMS}

We start this section with a result on stability of the linear equation.

Proposition 5.1. Assume that $\alpha \in L^{\infty}(\mathbb{R} / T \mathbb{Z})$ satisfies (4.1) and $\alpha \neq 0$. If (1.5) holds or $T<\tau(b)$, then (2.1) is asymptotically stable.

In these assumptions it follows from Propositions 4.1, 3.1 that the equation has no skew-periodic solutions. The next lemma will prove that there are also no $T$-periodic solutions. Then Proposition 5.1 can be proved using the same technique of Theorem 1 in [3].

Lemma 5.2. Assume that for some $\alpha \in L^{\infty}(\mathbb{R} / T \mathbb{Z}), \alpha \neq 0$, satisfying (4.1) there exist nontrivial $T$-periodic solutions of $(2.1)$. Then $b>\left(1+\zeta_{0}^{2}\right) c^{2} / 4$ and $T \geq \tau(b)+\pi / \delta$ where $\delta$ is given by (1.6).

Proof. Let $\phi(t)$ be a nontrivial $T$-periodic solution. Integrating over a period $\int_{0}^{T} \alpha(t) \phi(t) d t=0$. Then $\phi$ must change the sign. After a time translation it can be assumed that $\phi(0)=\phi(\tau)=\phi(T)=0$, for some $\tau \in[0, T]$ with $\phi^{\prime}(0)=\phi^{\prime}(T)=1, \phi^{\prime}(\tau)<0$. If, for example, $\phi^{\prime}(0) \leq-\phi^{\prime}(\tau)$, we apply Lemma 4.2 to conclude that $b>\left(1+\zeta_{0}^{2}\right) c^{2} / 4$ and $\tau \geq \tau(b)$. The Sturm comparison theory implies that $T-\tau \geq \pi / \delta$. 
Proofs of Theorems I and II. In the assumptions of Theorem I or II(i), one can use the same argument of [6, Theorem 1] together with Lemma 5.2 to deduce that (1.1) has a unique $T$-periodic solution $x(t)$. Let $b^{\prime}<b$ be such that $0<\partial_{x} g(t, x(t)) \leq b^{\prime}, t \in \mathbb{R}$. Since $\tau\left(b^{\prime}\right)>\tau(b) \geq T$, the asymptotic stability is a consequence of Proposition 5.1 and the principle of linearized stability. To prove Theorem II(ii) let $\sigma \in(0, T)$ be such that (3.1) is inversely unstable and let $\left\{\zeta_{n}\right\},\left\{\eta_{n}\right\}$ be sequences in $\mathbb{R}$ such that $g^{\prime}\left(\zeta_{n}\right) \rightarrow b, g^{\prime}\left(\eta_{n}\right) \rightarrow 0$. (It is possible since (1.7) holds.) Now let $\left\{x_{n}\right\}$ be a sequence of functions in $\mathbf{C}^{\infty}(\mathbb{R} / T \mathbb{Z})$ such that $x_{n}(t)=\zeta_{n}$ if $t \in(1 / n, \sigma-1 / n)$ and $x_{n}(t)=\eta_{n}$ if $t \in(\sigma+1 / n, T-1 / n)$. Then $g^{\prime}\left(x_{n}\right) \rightarrow b s_{\sigma}$ in $L^{\infty}$ weak * by the dominated convergence theorem. It follows from Lemma 2.1 that $\Delta\left[g^{\prime}\left(x_{n}\right)\right]<-(1+\exp (-c T))$ for large $n$. Then $p_{n}=x_{n}^{\prime \prime}+c x_{n}^{\prime}+g\left(x_{n}\right)$ satisfies (1.3) and $x_{n}$ is an unstable $T$-periodic solution of (1.1) for $p_{n}=p$. The proof of the existence of the second order subharmonic uses the same kind of degree argument found in [7, Theorem 4.2].

To finish the paper, we justify Remark 1 after Theorem II. If (1.1) has a second order subharmonic solution $x(t)$, then $y(t)=x(t+T)-x(t)$ is a skewperiodic solution of (2.1), where $\alpha \in L^{\infty}(\mathbb{R} / T \mathbb{Z})$ satisfies $\alpha(t)(x(t+T)-x(t))=$ $g(t, x(t+T))-g(t, x(t))$. In consequence, $T>\tau(b)$.

\section{REFERENCES}

1. L. Cesari, Asymptotic behavior and stability problems in ordinary differential equations, Springer-Verlag, Berlin, 1971.

2. L. K. Jackson, Existence and uniqueness of solutions of boundary value problems for Lipschitz equations, J. Differential Equations 32 (1979), 76-90.

3. A. C. Lazer and P. J. McKenna, On the existence of stable periodic solutions of differential equations of Duffing type, Proc. Amer. Math. Soc. 110 (1990), 125-133.

4. E. Lee and L. Markus, Foundations of optimal control theory, Wiley, New York, 1967.

5. W. Magnus and S. Winkler, Hill's equation, Dover, New York, 1979.

6. J. J. Nieto and L. Sanchez, Periodic boundary value problem for some Duffing equations, Differential and Integral Equations 1 (1988), 399-408.

7. R. Ortega, Stability of a periodic problem of Ambrosetti-Prodi type, Differential and Integral Equations 3 (1990), 275-284.

8. __ Topological degree and stability of periodic solutions for certain differential equations, J. London Math. Soc. 42 (1990), 505-516.

9. V. Pliss, Nonlocal problems of the theory of oscillations, Academic Press, New York, 1966.

10. R. A. Smith, Massera's convergence theorem for periodic nonlinear differential equations, J. Math. Anal. Appl. 120 (1986), 679-708.

11. T. Yoshizawa, Stability theory and the existence of periodic solutions and almost periodic solutions, Springer-Verlag, New York, 1975. SPAIN

Departamento de Matemática Aplicada, Universidad de Granada, 18071 Granada, 